\begin{tabular}{|c|c|c|}
\hline UÁQUIRI & PPG \\
\hline & Revista do Programa de Pós-Graduação em Geografia \\
UÁQUIRI - PPGGEO, v. 2, n. 1, p. 112 - 127, ano 2020 \\
Home page: https://periodicos.ufac.br/index.php/Uaquiri
\end{tabular}

\title{
O USO DE GEOPORTAIS BASEADOS EM SIG DURANTE A PANDEMIA DE CORONAVIRUS (COVID-19), COMO FERRAMENTA DE ACESSO ABERTO, DESTINADA AO CONTROLE E À TOMADA DE DECISÕES.
}

\author{
Dr. Rodrigo Otavio Peréa Serrano ${ }^{1,2^{*}}$, Me. Anderson Azevedo Mesquita ${ }^{1,3}$, Bel. Manoela Costa de \\ Araújo, Fábio da Silva Lúcio ${ }^{1}$, Gabriel Brandão Xavier ${ }^{2}$, Dr. José Genivaldo do Vale Moreira ${ }^{1,4}$ \\ ORCID: https://orcid.org/0000-0002-7786-8305; http://orcid.org/0000-0003-0947-8070; \\ https://orcid.org/0000-0001-9025-196X; https://orcid.org/0000-0002-7273-7648; \\ https://orcid.org/0000-0001-8071-1862; https://orcid.org/0000-0002-2994-8482
}

\begin{abstract}
${ }^{1}$ Universidade Federal do Acre (UFAC), Curso de Geografia Bacharelado, Rio Branco, Acre, Brasil; ${ }^{2}$ Professor do Programa de Pós-graduação em Ciência, Inovação e Tecnologia para a Amazônia da UFAC, Rio Branco, Acre, Brasil; ${ }^{3}$ Doutorando do Programa de Pós-graduação em Geografia da Universidade Federal de Rondônia, Porto Velho, Rondônia; ${ }^{4}$ Professor do Centro Multidisciplinar da UFAC, Campus Floresta, Cruzeiro do Sul,

Acre, Brasil.

*roperreas@gmail.com
\end{abstract}

Recebido em: 06/05/2020, Aceito em: 19/05/2020, Publicado em: 22/06/2020 DOI:

\section{RESUMO}

Em dezembro de 2019, diversos casos de uma síndrome respiratória aguda grave foram relatados na província de Hubei, Wuhan, China, com rápida transmissão para outras partes da Ásia e do mundo. Essa doença é conhecida como Covid-19 e apresenta impacto socioeconômicos ainda incalculáveis. A atual capacidade de atualização das informações em tempo real ao redor do mundo, permitiu a espacialização dos casos da doença e avaliação do risco de contágio, permitido uma rápida resposta dos órgãos governamentais. Dessa forma, este artigo apresenta uma serie de plataformas, baseadas em sistemas de informações geográficas (SIG), destinados ao mapeamento da pandemia de coronavírus. Por fim, concluiu-se que os métodos de espacialização se tornaram essenciais para fomentar, planejar e tomar decisão, no combate a uma pandemia. Além disso, tais plataformas, popularizaram o acesso a informação, através do compartilhamento dinâmico da ocorrência do covid-19 pelo mundo.

Palavras-chave: geografia dos riscos; síndrome respiratória; SARS-Co-V2

THE USE OF GIS-BASED GEOPORTALS DURING THE CORONAVIRUS PANDEMIC (COVID-19), AS AN OPEN ACCESS TOOL, INTENDED FOR CONTROL AND DECISIONMAKING.

\begin{abstract}
In December 2019, several cases of a severe acute respiratory syndrome were reported in Hubei province, Wuhan, China, with rapid transmission to other parts of Asia and the world. This disease is known as Covid-19 and has a still incalculable socioeconomic impact. The current ability to update
\end{abstract}


information in real time around the world has enabled the spatialization of cases of the disease and the assessment of the risk of contagion, allowing for a quick response from government agencies. Thus, this article presents a series of platforms, based on geographic information systems (GIS), aimed at mapping the coronavirus pandemic. Finally, it was concluded that spatialization methods have become essential to foster, plan and make decisions in the fight against a pandemic. In addition, such platforms have popularized access to information, through the dynamic sharing of the occurrence of covid-19 around the world.

Keywords: risk geography; respiratory syndrome; SARS-CoV2

\section{EL USO DE GEOPORTALES BASADOS EN SIG DURANTE LA PANDEMIA DE CORONAVIRUS (COVID-19), COMO UNA HERRAMIENTA DE ACCESO ABIERTO, PREVISTA PARA EL CONTROL Y LA TOMA DE DECISIONES.}

\section{RESUMEN}

En diciembre de 2019, se informaron varios casos de un síndrome respiratorio agudo severo en la provincia de Hubei, Wuhan, China, con transmisión rápida a otras partes de Asia y el mundo. Esta enfermedad se conoce como Covid-19 y tiene un impacto socioeconómico aún incalculable. La capacidad actual de actualizar la información en tiempo real en todo el mundo ha permitido la espacialización de los casos de la enfermedad y la evaluación del riesgo de contagio, lo que permite una respuesta rápida de las agencias gubernamentales. Por lo tanto, este artículo presenta una serie de plataformas, basadas en sistemas de información geográfica (SIG), destinadas a mapear la pandemia de coronavirus. Finalmente, se concluyó que los métodos de espacialización se han vuelto esenciales para fomentar, planificar y tomar decisiones en la lucha contra una pandemia. Además, tales plataformas han popularizado el acceso a la información, a través del intercambio dinámico de la ocurrencia de covid19 en todo el mundo.

Palabras clave: geografía de riesgo; síndrome respiratorio; SARS-CoV2

\section{INTRODUÇÃO}

Em dezembro de 2019, diversos casos de uma síndrome respiratória aguda grave (sigla em inglês "SARS"), posteriormente denominada de Covid-19 (SARS-Co-V2), foram relatados na província de Hubei, Wuhan, China, com rápida transmissão para outras partes da Ásia e do mundo (CCDC, 2020).

Para se ter ideia da gravidade e da velocidade de transmissão do vírus causador do Covid-19, 48 dias depois do primeiro caso diagnosticado, registros convergiam para quantidade superior 1000 casos, evidenciando uma velocidade de transmissão 10 vezes superior que o potencial de transmissão do MERS-Co-V 2012-2014, conhecida como a síndrome Respiratória do Oriente Médio, que levou aproximadamente, 2 anos e meio até alcançar o mesmo número de infectados. Adicionalmente o potencial transmissor de novo coronavírus é 2,5 vezes maior do que o SARS-Co-V1, que ocorreram entre 2012 a 2014. Essa velocidade despertou a atenção 
da Organização Mundial de Saúde (OMS), que declarou o surto de Covid-19, como uma emergência de saúde pública de interesse internacional (WHO, 2020).

Em revisão breve de literatura, observou-se vários estudos publicados em regime de urgência, na tentativa de divulgar, espacializar e explicar as métricas do comportamento de transmissão e proliferação, tanto em nível local, quanto global, levando em consideração a distribuição dos intervalos seriais, período de incubação e risco de disseminação internacional (BOGOCH et al., 2020; BOULOS and GERAGHTY, 2020; ZHAO et al., 2020). A disponibilização dos registros da doença, geograficamente espacializados em tempo real, ajudaram a comunicação e avaliação do risco de contágio e disseminação, especialmente durante o período de pandemia global (BOULOS and GERAGHTY, 2020; BROWNSTEIN and GERAGHTY, 2020; XU and KRAEMER, 2020).

A espacialização de ocorrências e focos epidemiológicos são fundamentais para as ações de combate, contenção e controle de doenças. Nesse sentido, Koch (2005) relata que o primeiro registro de mapas aplicados a área de saúde ocorreu em 1694, para auxiliar no controle de uma praga que atingiu a Itália. Nas últimas 5 décadas, após o surgimento dos sistemas informatizados, agilizou-se exponencialmente a capacidade mundial de processar informações geocodificadas, permitindo a espacialização, visualização, detecção e análises probabilísticas de riscos de proliferação de determinada doença.

A espacialização de ocorrências epidemiológicas emergente, pode facilitar o monitoramento e antecipar possíveis disseminações da infecção, desse modo, este artigo pretende apresentar uma séria de sites e aplicativos práticos de SIG on-line, destinados ao mapeamento da pandemia de coronavírus. Importante salientar que cada uma das plataformas analisadas, podem apresentar diferentes velocidades de atualização das informações, sujeitas a discrepância em números de infectados e óbitos registrados.

\section{METODOLOGIA.}

Foram usadas diferentes plataformas destinadas a geolocalização das ocorrências de coronavirus (Covid-19), quanto a facilidade de acesso, periodicidade de atualização e informações disponibilizadas ao usuário. Priorizou-se fontes oficiais e estruturas ligadas a grupos de pesquisas e/ou plataformas usadas em diferentes níveis da administração pública. 


\section{APRESENTAÇÃO E DISCUSSÃO DAS PLATAFORMAS GEOCODIFICADAS.}

Quando uma doença apresenta características de contágio por secreções de vias respiratórias, é possível contaminar diferentes superfícies como celular, mesas, maçanetas, brinquedos, teclados de computadores etc. Essas características permitem rápida expansão da doença pelo mundo, exigindo resposta rápida das diferentes instituições de controle epidemiológico. Dessa forma, plataformas que disponibilizam informações em tempo real, com painéis geoespacializados, contribuem sobremaneira para respostas efetivas ao problema (KOCH, 2005; BOULOS and GERAGHTY, 2020; BROWNSTEIN et al., 2020; XU and KRAEMER, 2020; WHO, 2020)

Em janeiro de 2020, já existiam sete geoportais entre os dez principais sites que solicitavam os serviços da Esri ArcGis Online, com mais de 160 milhões de visualizações (BOULOS and GERAGHTY, 2020). Tal volume de acesso, mostra como as pessoas estão ansiosas para rastrear ameaças reais a saúde, deixando evidente que qualquer cidadão com acesso à internet, pode acessar grande volume de informações sobre a doença em destaque.

A seguir apresenta-se alguns geoportais com informações com abrangência mundial, seguindo de geoportais sobre o continente americano, e do Brasil, fechando com um site que disponibiliza informações sobre covid19 no estado do Acre.

\section{a. Geoportal da Organização Mundial da Saúde (OMS)}

O geoportal de monitoramento do coronavírus da OMS é uma plataforma destinada a compartilhar as informações especializadas do avanço da pandemia (Figura 1). As informações apresentadas no geoportal resultam de uma vasta compilação de informações disponibilizados por site oficiais vinculados aos Ministérios da Saúde de vários países do mundo. Esse geoportal sobre covid19 foi apresentado em 26 de janeiro de 2020, usando a plataforma do ArsGIS Operations Dashboard (WHO, 2020). 
Figura 1: Geoportal da OMS atualizado em 20/04/2020, acessado em 26/04/2020 (WHO,2020).

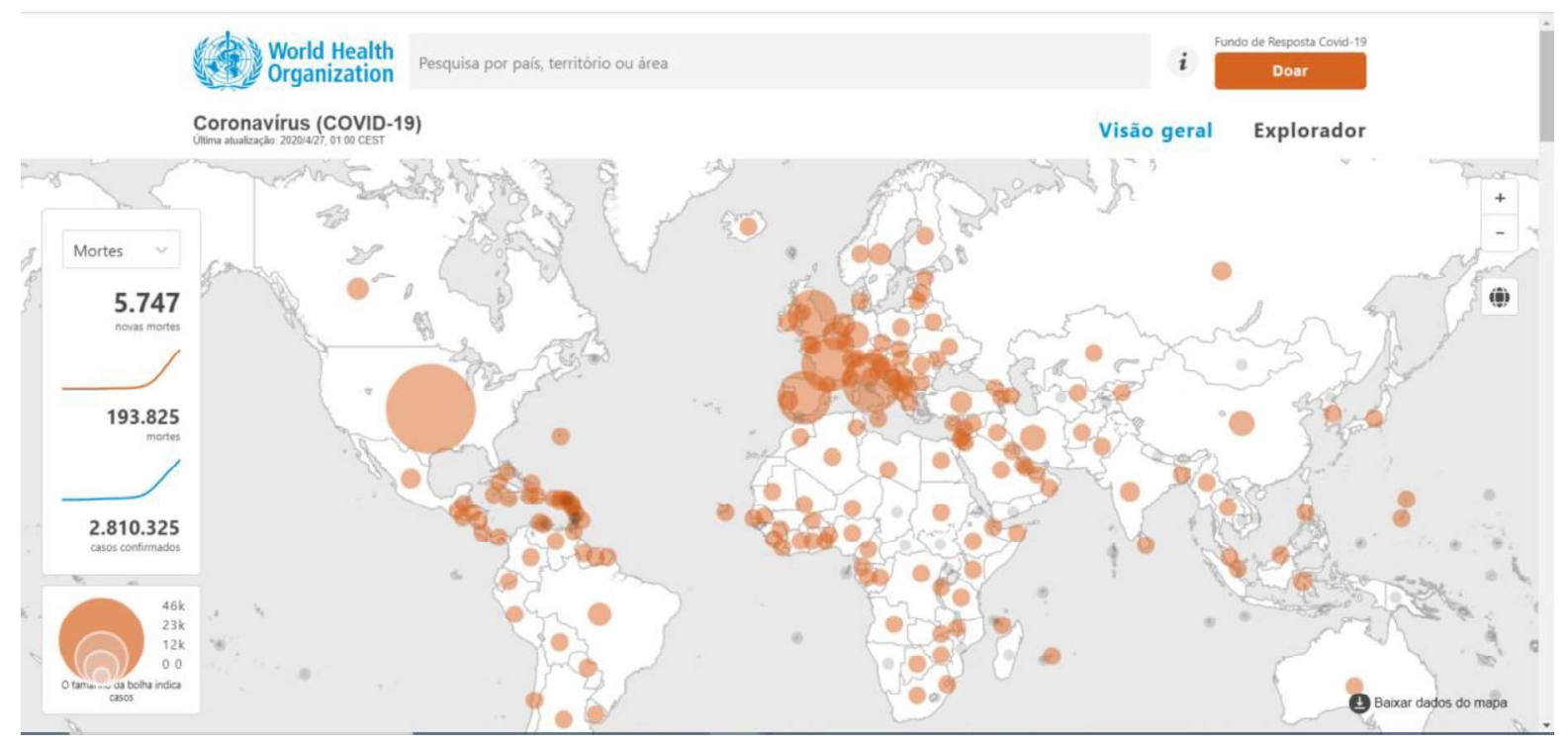

Link 1 - https://covid19.who.int/

A OMS dirige e coordena a saúde internacional, agrupando informações e traçando estratégias de resposta e combate as doenças transmissíveis. Sua plataforma utiliza um Sistema de Informação Geográfica (SIG), para acelerar as análises e tomadas de decisão, além da divulgação para internautas do mundo inteiro (WHO, 2020).

O geoportal da OMS, também disponibiliza curvas epidemiológicas dos casos confirmados e óbitos, além de gráficos comparativos entre diferentes países. A curva dos dados acumulados demostra a evolução da pandemia no mundo, porém esse tipo de gráfíco, com dados totais acumulados, não permite comparar a evolução entre os países, uma vez que cada um tem população e densidade populacional diferentes.

As informações sobre a covid-19 são atualizadas automaticamente por ArcGIS GeoEvent Serve várias vezes ao dia, correlacionando essas informações no espaço geográfico, permitindo um bom desempenho em até 12 níveis de zoom.

A facilidade de ter acesso às informações sobre a evolução da pandemia pelo coronavirus, beneficia diferentes grupos sociais, econômicos e intelectuais, e Segundo Boulos e Geraghty (2020), aproximadamente $8 \%$ dos usuários da plataforma escolheram a versão da plataforma destinada a aparelhos de telefonia móvel (smartfone) ou tablets, caminhando junto com a facilidade de acesso a informação na palma da mão. 


\section{b. Geoportal do Centro de Ciência e Engenharia de Sistemas da Johns Hopkins University.}

Os especialistas da Johns Hopkins em saúde pública global, doenças infecciosas e preparação para emergências estão desenvolvendo ações de resposta internacional a pandemia de Covid-19. O geoportal apresentam informações especializadas para ajudar no entendimento de propagação do vírus, informar ao público, além de orientar a tomada de decisão.

Em resposta a pandemia de coronavírus e o risco à saúde pública o Centro de Ciência e Engenharia de Sistemas (CSSE, sigla em inglês) da Johns Hopkins University, desenvolveu um geoportal para visualizar e rastrear em tempo real os casos de covid-19 em nível mundial. O site foi disponibilizado publicamente em 22 de janeiro de 2020, mostrando a localização dos casos confirmados (Figura 2).

O sistema de disponibilização das informações também é baseado na plataforma ArcGIS Online, que extrai os dados da OMS, do Centro de controle e Prevenção de Doenças (sigla em inglês "CDC") e de várias instituições oficiais de disponibilidade de dados sobre a pandemia de covid-19.

Figura 2: Geoportal da Painel COVID-19 do Centro de Ciência e Engenharia de Sistemas (CSSE) da Universidade Johns Hopkins (JHU) (CSSE, 2020)

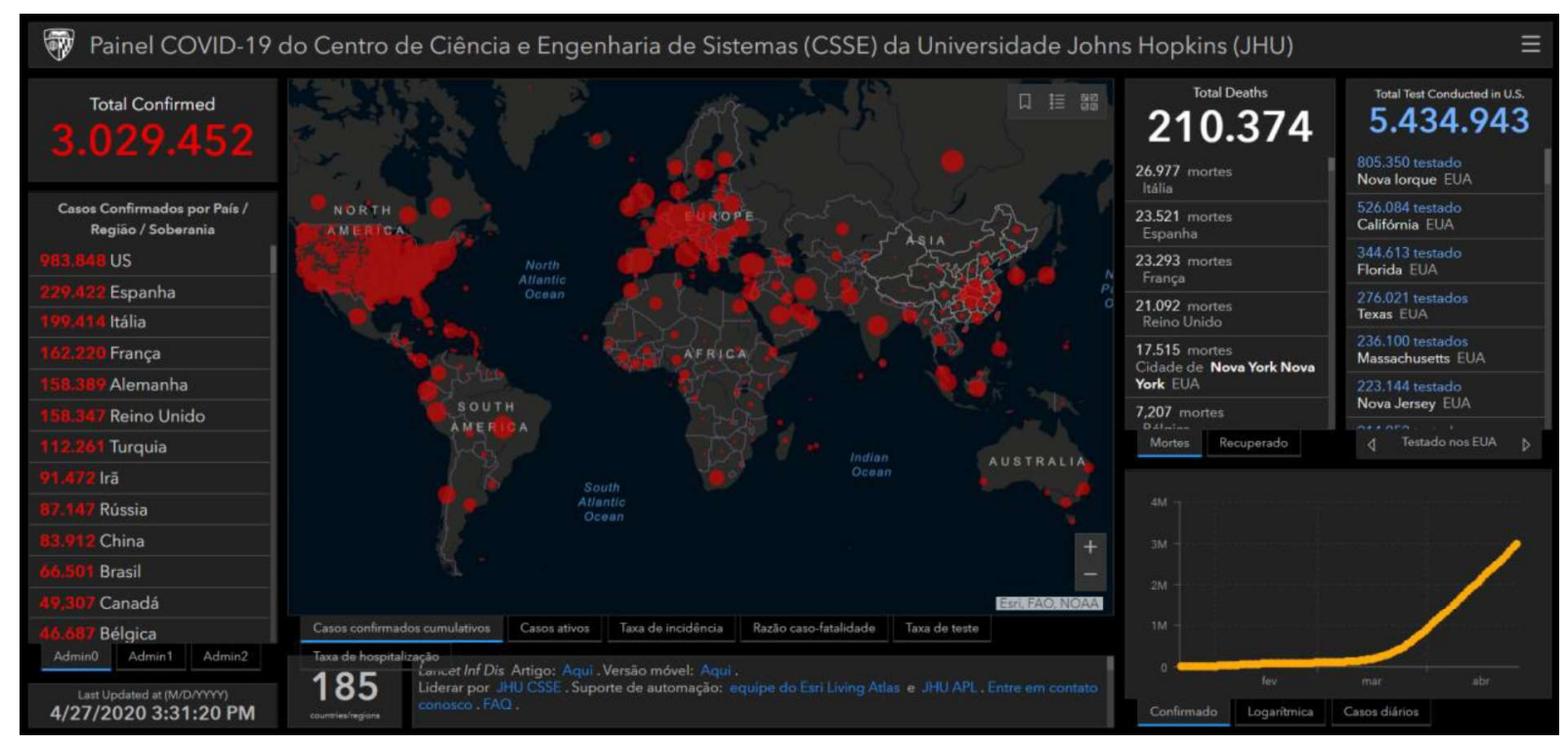

Link 2 - https://www.arcgis.com/apps/opsdashboard/index.html\#/bda7594740fd40299423467b48e9ecf6 
O mapa interativo desse geoportal, localiza e registra os casos confirmados, óbitos e recuperações confirmadas. Apresentado gráficos da propagação e evolução do vírus ao longo do tempo, permitindo acesso ao dia e a hora da atualização, além das fontes de dados mais

relevantes (GARDNER, 2020). O autor relata ainda, a dificuldade de se coletar dados em uma resolução espacial fina, a nível de cidade, distrito ou bairro, sendo essas as informações as mais buscadas. As ferramentas comumente conhecidas de geolocalização em plataformas móveis, poderiam ajudar nesse detalhamento, porém, necessitariam de autorização de compartilhamento da localização, além do compartilhamento do diagnóstico de covid-19.

Infelizmente esse geoportal não possui os serviços de arquivamento para visualizar a retrospectiva completa dos dados espacializados. Para se ter ideia da evolução da pandemia ao longo do tempo, o site só disponibiliza um gráfico da evolução temporal, dos dados confirmados, total de recuperados e número de óbitos. No entanto, é extremamente útil por apresentar uma atualização mundial de casos, em tempo real, mostrando a situação pandêmica em diferentes regiões do planeta.

\section{c. Geoportal da Microsoft}

O site disponibilizado pela Microsoft, monitora a progressão dos casos registrados de coronavírus pelo mundo, permitindo a pesquisa por país, e no caso do Brasil, disponibiliza as informações por Estado. É uma plataforma auto explicativa com mapas semelhantes aos da OMS, com o diferencial de mostrar as informações separadas de cada país, clicando-se na área do mapa ou na lista de países é disponibilizada. Além disso, o site exibe uma lista de notícias, vídeos e gráficos interativos sobre o avanço do coronaviros por região, disponibilizando os dados ativos, óbitos e recuperados (MICROSOFT, 2020). A figura (03) representa o layout do geoportal da Microsoft acessado no dia 27 de abril de 2020

Essa plataforma fica hospedada no Bing, serviço rival do Google, e rastreia as ocorrências de infecção por Covid-19, através das informações disponibilizadas pela OMS, pelo Centro de Prevenção e Controle de Doenças dos Estados Unidos (CDC), pelo Centro Europeu de Controle e Prevenção de Doenças, entre outros. 
Figura 3: Geoportal da Microsoft (MICROSOFT, 2020)

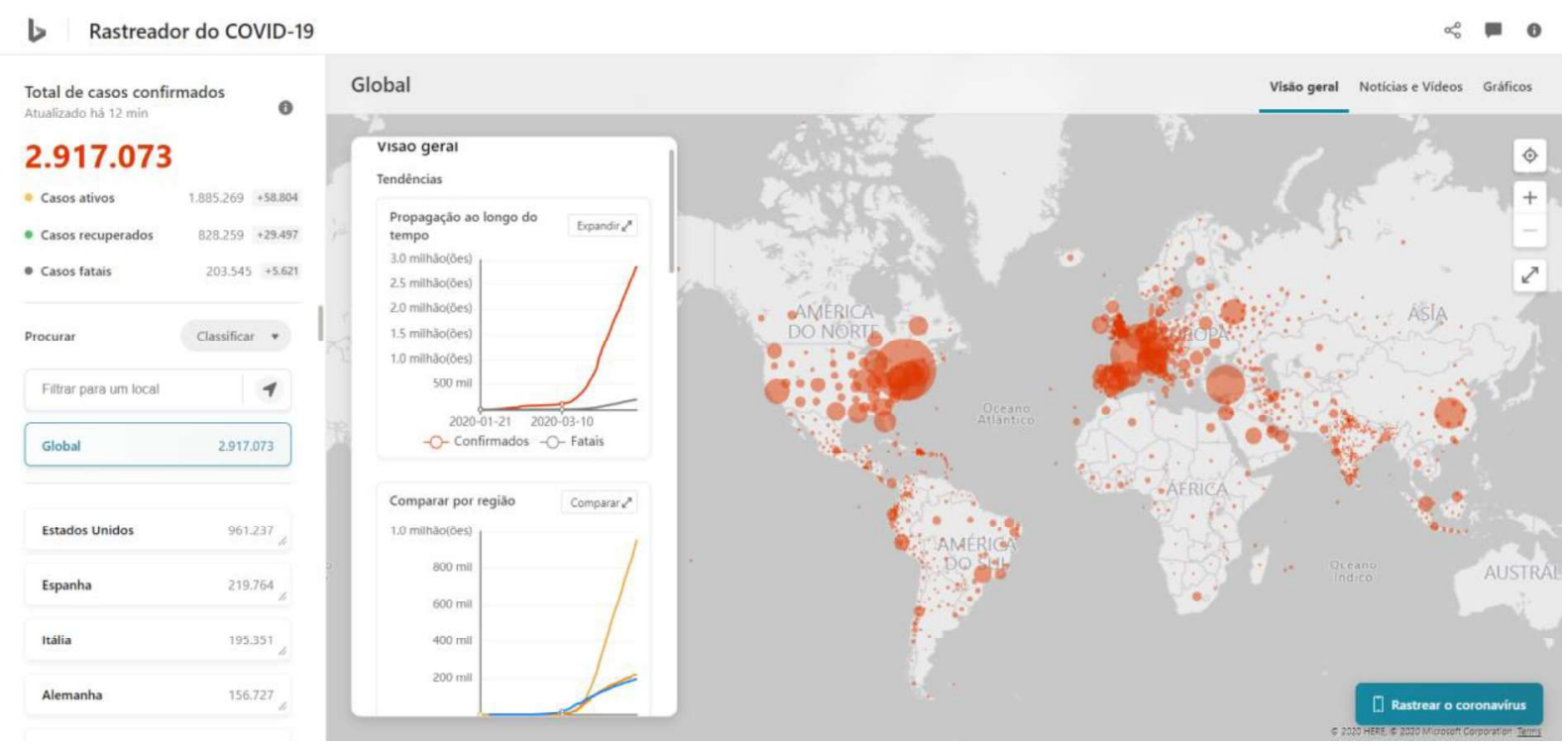

Link 3 - https://www.bing.com/covid

Como esses mapas são gerados com dados proveniente de varredura sistêmica da rede mundial de computadores, acaba que em muitas situações apresentam dados mais atualizados que os sites oficiais de cada país. Essa diferença ocorre, em decorrência da periodicidade da atualização dos dados oficiais

Observa-se também, defasagem dos dados em ralação aos dados disponibilizados pelo Ministério da Saúde do Brasil, decorrente da menor frequência diária de atualização dos dados. Um exemplo desse lapso temporal foi o registro oficial do primeiro óbito no Brasil, que ocorreu na terça feira dia 17 de março de 2020, foi divulgado pela mídia por volta das 11 horas e entrou no registro do Bing em menos de uma hora, entretanto, o site oficial do coronavírus Brasil (https://covid.saude.gov.br/) do ministério da Saúde, confirmou somente as 17 horas do mesmo dia.

\section{d. Geoportal da Pan American Health Organization (PAHO)}

Esse geoportal foi estruturado pela PAHO, juntamente com a Organização Mundial da Saúde (OMS), sendo resultado de parcerias com a plataforma ArcGIS Web Application que, juntos apresentam os casos cumulativos da COVID-19 relatados por países e territórios nas Américas (Figura 04) 
Figura 4: Geoportal apresentados os casos acumulados nas Américas (PAHO, 2020)

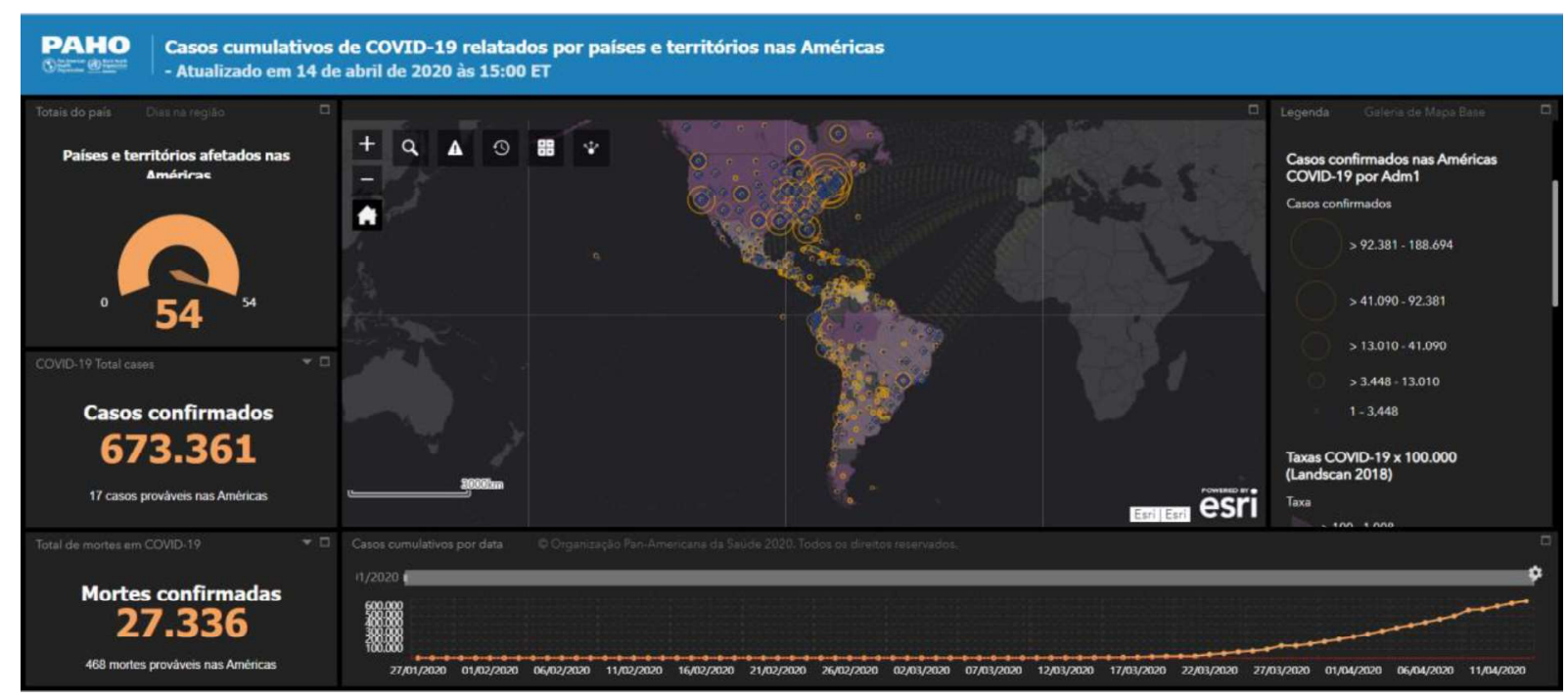

Link 4 - https://who.maps.arcgis.com/apps/webappviewer/index.html?id=2203b04c3a5f486685a15482a0d97a87\&extent=$\underline{20656313.6818 \% 2 \mathrm{C}-3596894.4332 \% 2 \mathrm{C} 1611932.8945 \% 2 \mathrm{C} 7390469.7606 \% 2 \mathrm{C} 102100 \& \text { site }=\text { paho }}$

Esse geoportal apresenta informações quantitativas do número de casos diagnosticados e de óbitos confirmados acumuladamente. O mapa destaca também a intensidade de pessoas infectadas em diferentes regiões do continente americano.

O gráfico apresentado no geoportal da PAHO, disponibiliza uma série histórica desde o primeiro caso confirmado nas américas, datado de 27/01/2020, seguida da séria acumulada com 2 dias de defasagem na atualização dos dados.

Além das informações geoespacializadas, apresenta um link para página da PAHO destinada a emergências em saúde com diversas informações detalhas para o combate e controle do coronavírus (PAHO, 2020b)

\section{e. Geoportal Coronavirus Brasil}

Esse geoportal foi criado pelo Ministério da Saúde do Brasil, com o objetivo de manter a população informada sobre evolução da pandemia de Covid-19 no Brasil. O painel apresenta informações espacializadas, permitindo uma análise da evolução de contágio do vírus ao longo do tempo, além de gráficos de dados acumulados apontando a curva epidêmica da doença (Figura 5). 
Figura 5: Geoportal de monitoramento pandêmico de covid-19 no Brasil (MS. BRASIL, 2020).

CORONAVÍRUS // BRASIL

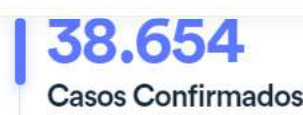

Painel Geral SRAG Insumos
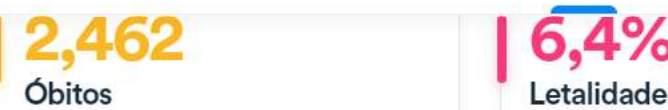

Letalidade

Casos por região

Casos no Brasil

Confirmados Óbitos

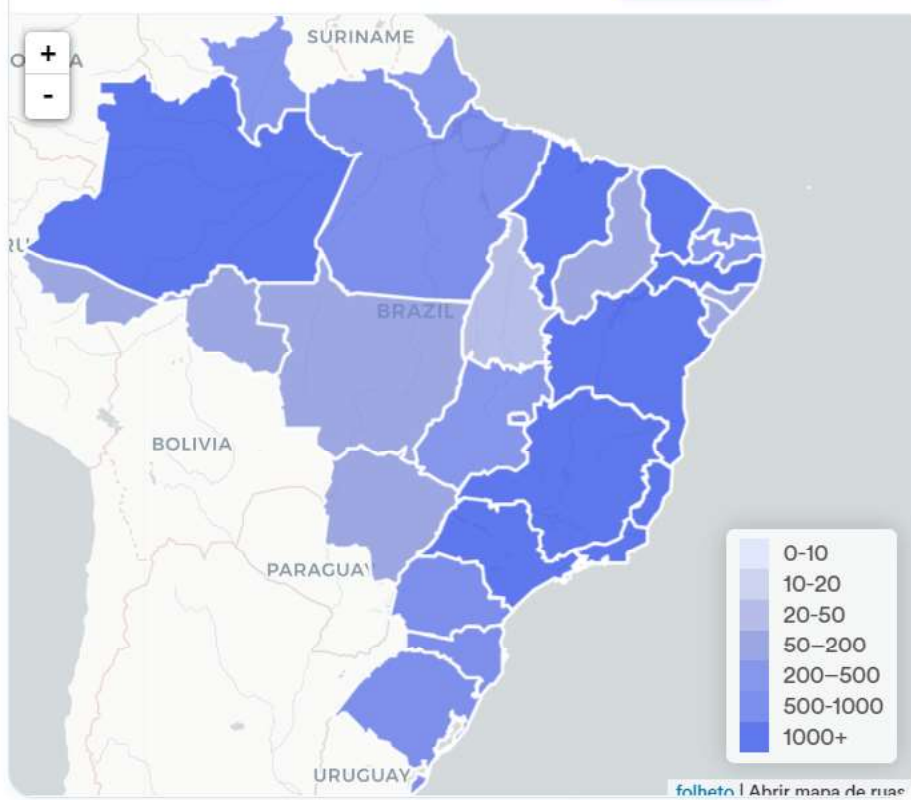

Norte

Nordeste

Centro-Oeste

Sudeste

Sul

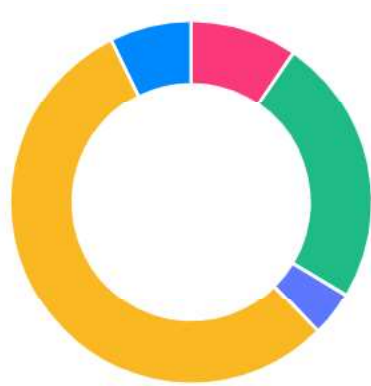

$36919,5 \%$

$9300 \quad 24,1 \%$

$15624,0 \%$

$21285 \quad 55,1 \%$

$2816 \quad 7,3 \%$

Link 5 - https://covid.saude.gov.br/

Além de informar o número de casos confirmados e número de óbitos atualizados diariamente às 17 horas, o site também disponibiliza informações sobre Síndrome Respiratória Aguda Grave (SRAG), ocasionados pelo Covid-19 (SARS-CO-V2), Influenza A e B e casos em investigação.

Outras informações importantes, é a disponibilização do histórico dos casos hospitalizados por Síndrome Respiratória Aguda Grave (SRAG) em 2020, em comparação aos dados registrados em 2019, permitindo acompanhar a evolução em comparação a uma anos de referência (Figura 6). 
Figura 6: hospitalizado por semana epidemiológica (MS. BRASIL, 2020)

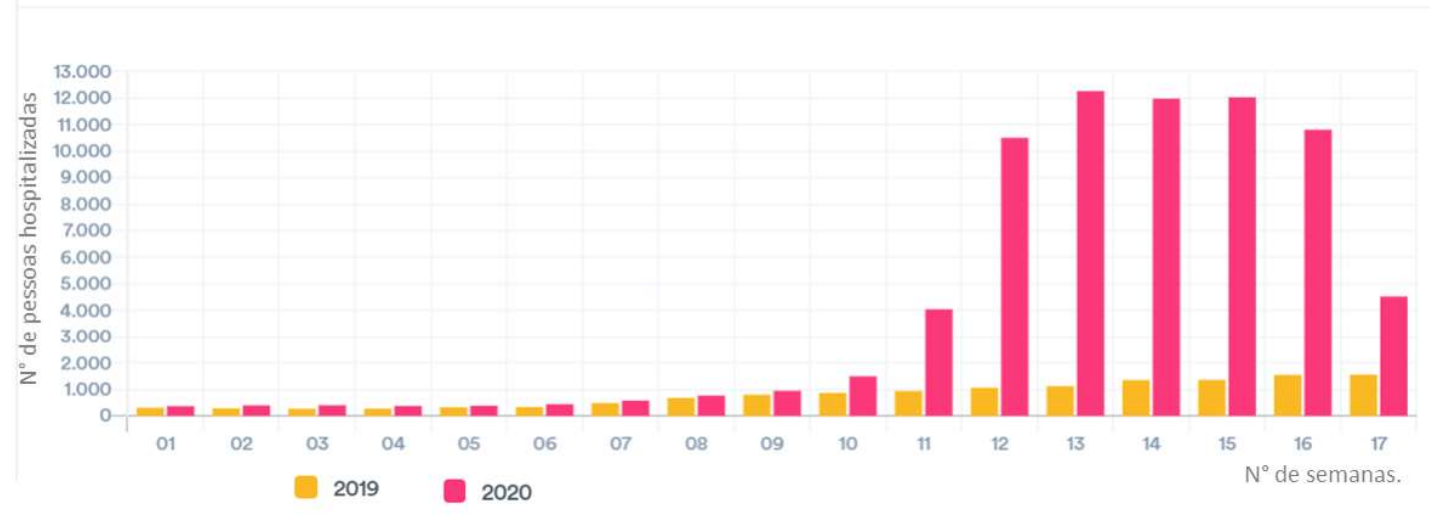

Link 6 - $\underline{\text { https://covid.saude.gov.br/ }}$

As informações de comparativo anual permitem analisar cenários incrementados por suspeitos de infecção pelo coronavírus mas, que por motivos alheios, não são submetidos ao teste de confirmação. Tal estimativa pode se basear na diferença entre os internados no ano de 2019 e em 2020.

\section{f. Geoportal Covid Radar Brasil}

Essa plataforma foi desenvolvida em parceria com diferentes instituições privadas. Ela reúne diversos aplicativos que disponibilizam grande quantidade de informações, para população. O intuito e colaborar com as ações de controle à doença, cuja colaboração é de uma base robusta e organizada dos dados referentes a mais de mil secretarias espalhadas pelo Brasil.

As informações são atualizadas em tempo real, permitindo a simulação do avanço do novo coronavírus por regiões, cidades e estados, e utiliza dados de mobilidade e propagação disponibilizados por fontes oficiais. A figura (07) representa o layout do geoportal do Covid Radar Brasil acessado no dia 27 de abril de 2020. 
Figura 7: Opções de monitoramento em tempo real do Painel covid radar (MS. BRASIL, 2020)

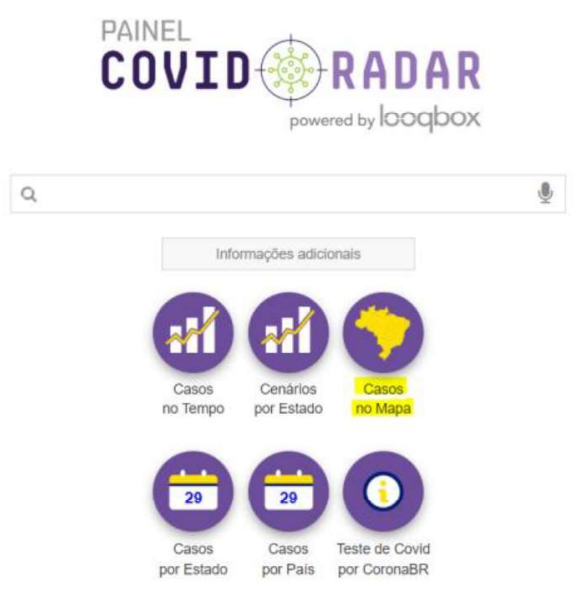

Link 7 - https://painel.covidradar.org.br/\#/

Conforme indicado na figura (07), a opção "casos no mapa", direciona para um mapa interativo, com o número de casos por milhão de habitantes, sendo o único a levar em consideração esse tipo de relação, permitindo uma comparação da real situação entre diferentes estados (Figura 8).

Figura 8: Mapa espacializados da ocorrência de covid 19 no Brasil [casos por milhão] (MS. BRASIL, 2020).

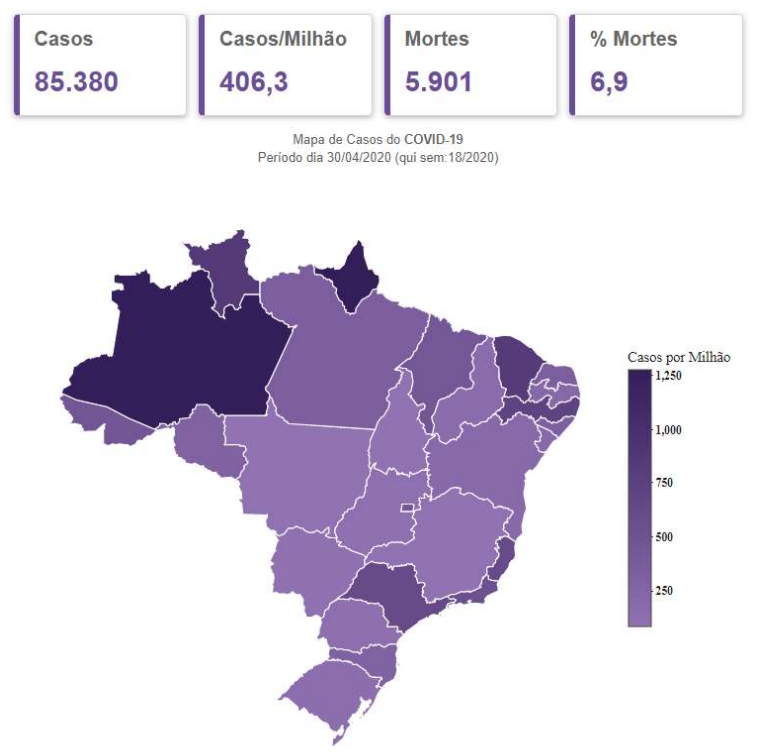

Fonte dos dados: Ministério da Saúde

Link 8 - https://painel.covidradar.org.br/\#/ 
Observa-se na Figuras (8 e 9), que levando em consideração a relação por milhão, o Estado do Amazonas e Amapá, com 5.254 e 1.080 casos confirmados respectivamente, estão em situação mais crítica, mesmo apresentando um número total de infectados inferiores aos estados de São Paulo e Rio de Janeiro, com 28.698 e 9.453 casos confirmados respectivamente, tendo como referência o dia 30/04/2020 (MS. Brasil, 2020).

Figura 9: Comparação do número de casos confirmados por milhão (MS. BRASIL, 2020)

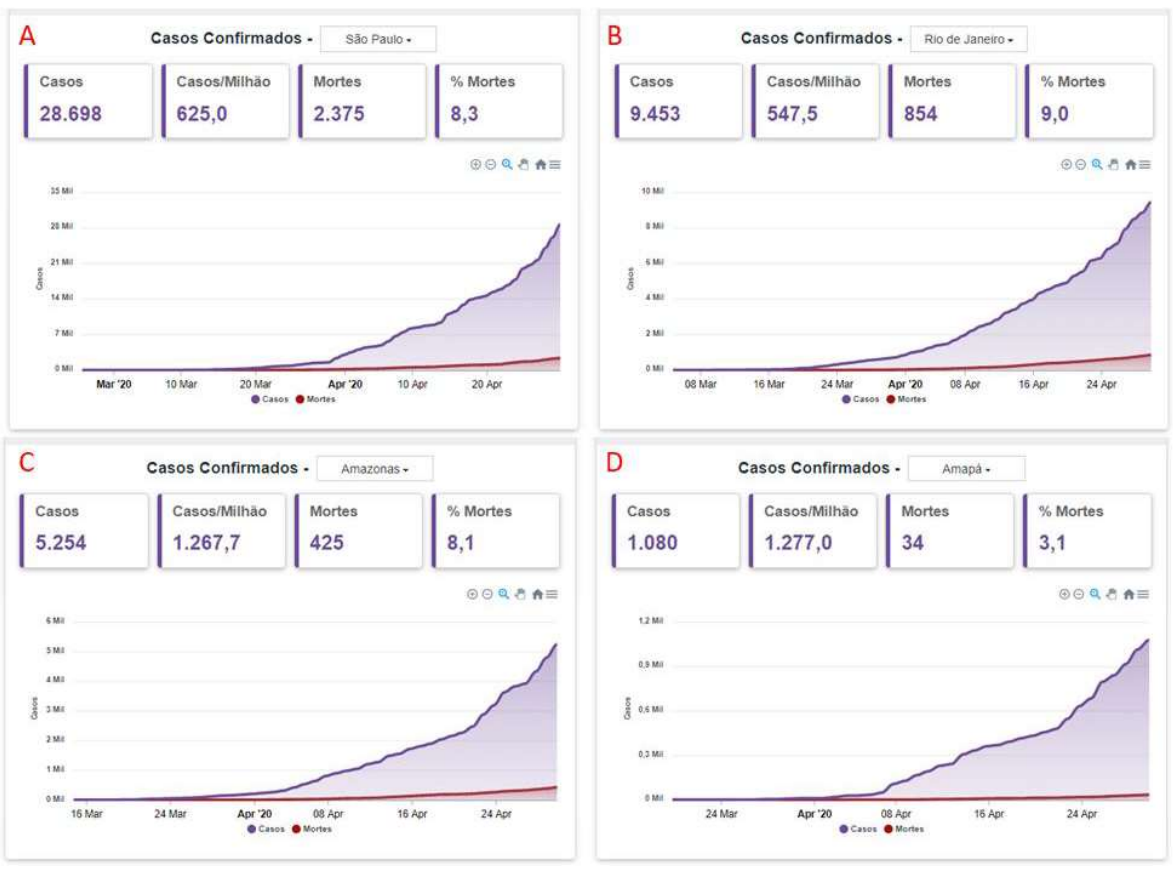

Além das informações espacializados e gráficos evolutivos da infecção ocasionadas pelo covid-19, o site também disponibiliza cenários por estado, levando em consideração os modelos utilizados na Alemanha e na Espanha, que adotaram medidas de isolamento em períodos diferentes durante a pandemia. Com base nesses senários e possível identificar a data provável dos picos epidemiológicos em todos os estados brasileiros, como base nos dados já disponibilizados.

\section{g. Outras maneiras pelas quais as tecnologias GIS podem ajudar no combate a epidemias.}

Informações como a localização de unidades de saúde destinadas ao tratamento das doenças, também podem agilizar a busca por tratamentos. Nesse sentido o APP Coronavírus 
SUS Brasil, atende tal anseio e, adicionalmente, oferece outras informações de prevenção e contenção de doenças associadas ao vírus.

Para evitar a falta de medicamentos e suprimentos, durante a emergência pandêmica é importante o mapeado os possíveis fornecedores de insumos necessários ao enfrentamento, bem como as rotas e sistemas de transporte. Também é importante mapear as diferentes indústrias que possam ter suas linhas de produção adaptadas para cobrir a deficiência de fornecimento das indústrias localizadas em locais afetados pela pandemia, assim como ocorreu com o fechamento das fabricas chinesas durante a epidemia de covid-19 naquele país.

A escassez de equipamentos hospitalares de segurança tornou as equipes profissionais mais vulneráveis, em todo o mundo. A perda de profissionais tanto por afastamento para tratamento quanto por mortes ocasionou a diminuição da oferta dos serviços especializados. Dessa forma, mapas digitais da cadeia de suprimentos são fundamentais para planejar e garantir o fornecimento mundial em caso de pandemia.

\section{CONSIDERAÇÕES FINAIS}

Os métodos de análise de dados espacializados estão se tornando cada vez mais essenciais para fomentar o planejamento e a tomada de decisão sobre os serviços de saúde, bem como alertar a população sobre medidas essenciais. No entanto, no campo da prestação de serviços para pessoas com deficiência e idosos, os métodos espaciais ainda apresentam limitações, que precisarão ser vencias ao longo do tempo.

As plataformas aqui apresentadas, fornecem uma estrutura espacial que permite identificar áreas prioritárias aos cuidados de saúde em tempos de crise. Mostrando, que independente de ser o civid-19, tal metodologia pode ser aplicada amplamente para identificar regiões vulneráveis a propagação de surtos epidemiológicos. Tais sistemas também podem indicar as regiões com necessidade de ações paliativas, com a intenção de deter a propagação de uma doença infecciosa, antes do primeiro caso na localidade.

Os modelos de SIG aplicados a epidemiologia aqui apresentados, se concentram em ferramentas baseadas em Web, mostraram-se importantes ferramentas de compartilhamento de dados e informações, em tempo real, capaz de apoiar na tomada de decisões em momentos de crise, como se mostrou diante das ações frente à pandemia por coronavírus. 
Por fim, tais plataformas caíram em uso popular, proporcionado informações em tempo real para diferentes classes sociais e culturais, compartilhando conhecimento da dinâmica de disseminação do SARS-Co-v2.

\section{REFERÊNCIAS}

BOGOCH I. I.; WATTS, A.; THOMAS-BACHLI, A.; HUBER, C.; KRAEMER, M. U. G.; KHAN, K. Pneumonia of unknown etiology in Wuhan, China: potential for international spread via commercial air travel. J Travel Med, p. 1-3, 2020. DOI: https://doi.org/10.1093/jtm $\underline{\text { taaa008. }}$.

BOULOS, M. N. K AND GERAGHTY, E. M. Geographical tracking and mapping of coronavirus disease COVID-19/severe acute respiratory syndrome coronavirus 2 (SARS-CoV-2) epidemic and associated events around the world: how 21 st century GIS technologies are supporting the global fight against outbreaks and epidemics. International Journal of Health Geographics, v. 18, n. 19, p. 1-18, 2020. DOI: https://doi.org/10.1186/s12942-020-00202-8

BROWNSTEIN, J.S.; FREIFELD, C.; MADOFF, L.C. Digital Disease Detection - Harnessing the Web for Public Health Surveillance. N. Engl. J. Med, n. 360, p. 2153-2157, 2009. DOI: https://doi.org/10.1056/NEJMp0900702.

CHINESE CENTER FOR DISEASE CONTROL AND PREVENTION (CCDC). The Epidemiological Characteristics of an Outbreak of 2019 Novel Coronavirus Diseases (COVID19)-China. 2020. Disponível em: http://weekly.chinacdc.cn/en/article/id/e53946e2-c6c4-41e99a9b-fea8db1a8f51. Acesso em: 13 abr. 2020.

GARDNER L. Mapping 2019-nCoV. Johns Hopkins, 2020. 1disponivel em: < https://systems.jhu.edu/research/public-health/ncov/ Acesso em: 03 maio 2020.

JOHNS HOPKINS (CSSE). Corona Virus 2019-nCoV Cases (The Living Atlas), 2020. Disponível em: https://livingatlas.arcgis.com/en/browse/\#d=2\&q=\%22Corona $\% 20$ Virus $\% 202019 \% 20 \mathrm{nCoV}$ \%20Cases\%22. Acesso em: 03 maio2020.

$\mathrm{KOCH}, \mathrm{T}$. Mapping the Miasma: Air, Health, and Place in Early Medical Mapping. cartographic perspectives, n. 25, p. 4-27, 2005. DOI: https://doi.org/10.14714/CP52.376

Microsoft, Microsoft Big Maps e MapPoit Web Service. Rastreador COVID-19, 2020. Disponível em: https://www.bing.com/covid. Acesso em: 26 abr 2020.

MINISTÉRIO DA SAÚDE (MS. Brasil). Covid Radar: Todos juntos contra a covid-19. Coletivo de mais de 40 organizações, 2020. Disponível em: https://www.covidradar.org.br/. Acesso em: 01 maio2020.

PAN AMERICAN HEALTH ORGANIZATION (PAHOa). Cumulative COVID-19 cases reported by countries and territories in the Americas, 2020. Disponível em: 
https://who.maps.arcgis.com/apps/webappviewer/index.html?id=2203b04c3a5f486685a15482 a0d97a87\&extent $=-20656313.6818 \% 2 \mathrm{C}-$ 3596894.4332\%2C1611932.8945\%2C7390469.7606\%2C102100\&site=paho. Acesso em: 15 abr. 2020.

PAN AMERICAN HEALTH ORGANIZATION (PAHOb). COVID-19 Situation in the Region of the Americas. Situation as of 14 April 2020 (14:00 EST). Disponível em: https://www.paho.org/en/topics/coronavirus-infections/coronavirus-disease-covid-19. Acesso em: 15 abr. 2020.

WORLD HEALTH ORGANIZATION (WHO/OMS). Statement on the second meeting of the International Health Regulations Emergency Committee regarding the outbreak of novel coronavirus (2019-nCoV), 2020. Disponível em https://www.who.int/news-room/detail/30-012020-statement-on-the-second-meeting-of-the-international-health-regulations-(2005)emergency-committee-regarding-the-outbreak-of-novel-coronavirus-(2019-ncov) Acesso em: 03 maio 2020.

XU, B.; KRAEMER M. U. G. Open access epidemiological data from COVID-19 outbreak. The Lancet: Infectious Diseases, Published: February 19, 2020. DOI: https://doi.org/10.1016/S1473-3099(20)30119-5

ZHAO, S.; LIN, Q.; RAN, J.; MUSA, S. S.; YANG, G.; WANG, W.; LOU, Y.; GAO, D.; YANG, L.; HE, D.; WANG, M. H. Preliminary estimation of the basic reproduction number of novel coronavirus (2019-nCoV) in China, from 2019 to 2020: A data-driven analysis in the early phase of the outbreak. International Journal of Infectious Diseases, n. 92, p. 214-217, 2020. DOI: https://doi.org/10.1016/j.ijid.2020.01.050 